\title{
Influence of [0001] tilt grain boundaries on the destruction of the quantum Hall effect in graphene
}

\author{
Anders Bergvall, ${ }^{1}$ Johan M. Carlsson, ${ }^{2}$ and Tomas Löfwander ${ }^{1, *}$ \\ ${ }^{1}$ Department of Microtechnology and Nanoscience-MC2, Chalmers University of Technology, SE-412 96 Göteborg, Sweden \\ ${ }^{2}$ Dassault Systèmes BIOVIA GmbH, Hohenzollernring 84, D-50672 Cologne, Germany \\ (Received 2 August 2014; revised manuscript received 1 June 2015; published 18 June 2015)
}

\begin{abstract}
The half-integer quantum Hall effect (QHE) is often suppressed in graphene grown by chemical vapor deposition on metals. The reason behind the suppression is unclear, and we hypothesize that it might be connected to extended defects in the material. In this paper we present results for the quantum Hall effect in graphene with [0001] tilt grain boundaries connecting opposite sides of Hall bar devices. Such grain boundaries contain 5-7 ring complexes that host defect states that hybridize to form bands with varying degree of metallicity depending on grain-boundary defect density. In a magnetic field, edge states on opposite sides of the Hall bar can be connected by the defect states along the grain boundary. This destroys Hall resistance quantization and leads to nonzero longitudinal resistance. Anderson disorder can partly recover quantization, where current instead flows along returning paths along the grain boundary depending on defect density in the grain boundary and on disorder strength. Since grain sizes in graphene made by chemical vapor deposition are usually small, this may help explain why the quantum Hall effect is usually poorly developed in devices made of this material.
\end{abstract}

DOI: 10.1103/PhysRevB.91.245425

PACS number(s): 73.50.Jt, 72.80.Vp, 85.75.Nn

\section{INTRODUCTION}

The half-integer quantum Hall effect (QHE) in monolayer graphene $[1,2]$ grown on silicon carbide $(\mathrm{SiC})$ substrates has been observed to metrological accuracy [3-7]. Very high breakdown currents have been recorded, and quantization remains accurate also at elevated temperatures. This material may therefore be the next choice for an improved resistance standard. On the other hand, QHE plateaux have not been measured to the same level of accuracy on Hall bars made of graphene grown by chemical vapor deposition (CVD) on metal substrates [8-10]. Since the electronic mobility in CVD graphene is not worse than that in graphene grown on $\mathrm{SiC}$ [11], the difficulty to observe the quantum Hall plateaux with very high precision is currently debated.

As is well known, the QHE is topologically protected in the sense that counterpropagating edge states are located on opposite sides of the Hall bar. Backscattering and concomitant breakdown of quantization therefore require these states, located far away from each other in the sample, to be connected. Any mechanism able to connect them suffices, but it is important for each quantum Hall system to identify and study in detail the dominant mechanisms. For graphene grown by CVD on metal substrates, the QHE breakdown may be due to extrinsic effects, such as defects and inhomogeneity introduced in the process of graphene transfer to insulating substrates used for devices (wrinkles or charge puddles), or to defects in the material itself, such as grain boundaries that usually are found in graphene made by CVD (see, for instance, the reviews in Refs. $[12,13])$.

In a recent experiment [10], it was indeed argued that grain boundaries may be the source of reduced quantization in devices made of CVD graphene. A clear theoretical picture of how the QHE is destroyed in graphene with grain boundaries is, however, still lacking. One particular and very special type

\footnotetext{
*tomas.lofwander@chalmers.se
}

of grain boundary has been the focus of works published before $[10,14,15]$. The grain boundary consists of a perfect row of 5-8-5 ring complexes that separates two perfect armchair ribbons oriented along the same axis. To join the armchair ribbons to the grain boundary, the ribbons are cut at $90^{\circ}$ to their armchair edges so that perfect zigzag edges are formed. These zigzag edges can be attached to the grain boundary. In a magnetic field, a picture appears of current flowing along an armchair edge in the ribbon and along a zigzag edge along the grain boundary over to the opposite edge of the ribbon where the current can flow back in the opposite direction. This special type of grain boundary is not the only or typical grain boundary in graphene [12,13,16-19], and a more extensive and systematic investigation of other grain boundaries is called for.

Here, we report a systematic investigation of the influence of [0001] tilt grain boundaries on the QHE in graphene. We show by numerical simulation that electronic states [20-24] at dislocation cores (5-7 ring complexes), which form several one-dimensional metallic bands along the grain boundary, can, in a strong externally applied magnetic field, connect two edge states on opposite sides of the Hall bar and thereby destroy quantization. The resulting conductance fluctuations depend on the number of dislocation cores in the grain boundary, which is related to the grain-boundary tilt angle and its physical length. This is similar to the situation for graphene grown on silicon carbide substrates, where it has been shown by numerical simulation [25] and experiment [26] that bilayer stripe defects connecting Hall bar edges destroy the QHE in that material. Only in samples without such large bilayer defects may one expect the precise quantization reported in Ref. [5].

\section{MODEL}

The grain-boundary models were constructed by the coincidence site lattice (CSL) theory according to the method presented in Ref. [27]. The CSL grain boundaries are 
(b)

$\Sigma=19$

(a)
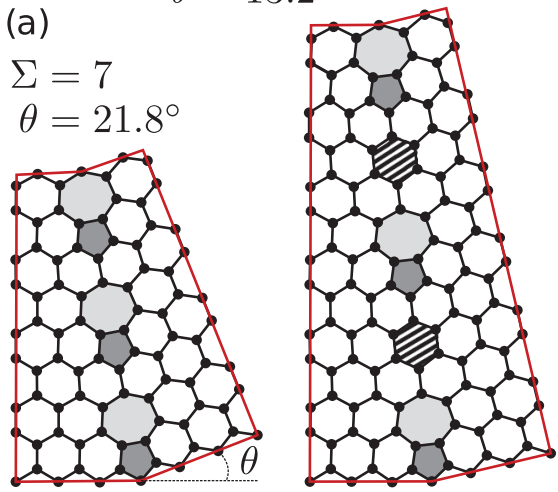

(c) $\Sigma=37$

$\theta=9.4^{\circ}$

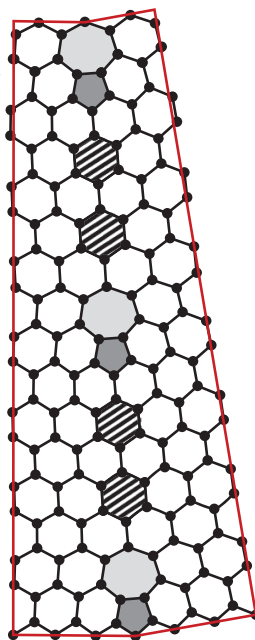

FIG. 1. (Color online) The first three in a series of grain boundaries (Table I) with decreasing misorientation angle $\theta$. The grain boundaries contain 5-7 ring defects on a line with an increasing number of hexagons (hatched) between neighboring defects.

characterized by the size of the CSL cell in terms of the unit cell $\Sigma=\Omega_{\text {CSLcell }} / \Omega_{\text {unitcell }}$, the misorientation angle $\theta$, and the period length along the grain boundary $d_{\text {rel }}$. We focus on a series of grain boundaries for which the Burger's vector is one lattice vector long $\left(n_{d}=1\right.$ series in Ref. [27]), meaning that we limit ourselves to a class of [0001] tilt grain boundaries with one 5-7 ring complex per grain-boundary unit cell but an increasing number of hexagons per unit cell for decreasing grain-boundary angles. This means that the distance between 5-7 ring defects increases by one hexagon as we move along the $n_{d}=1$ series towards smaller tilt angles (see Fig. 1). We consider the first six grain boundaries in the series, which we enumerate by $m=1,2, \ldots, 6$, and the corresponding $\Sigma$ values and misorientation angles are specified in Table I. The geometry was optimized by a force field calculation using the Dreiding force field as implemented in the FORCITE module in MATERIALS STUDIO [28]. We aim to model graphene grown on a substrate, so the graphene sheet was kept flat even at the grain boundaries by fixing the atomic relaxation perpendicular to the sheet during the relaxation. Using the atomic positions of the relaxed grain boundary, we create a two-terminal zigzag nanoribbon of varying width and study electron transport in a magnetic field.

TABLE I. List of CSL grain boundaries studied in the present paper, classified according to the unit-cell size $\Sigma$, grain-boundary misorientation angle $\theta$, and periodic length $d_{\text {rel }}$.

\begin{tabular}{lrrc}
\hline \hline$m$ & $\Sigma$ & $\theta$ & $d_{\text {rel }}(\mathrm{nm})$ \\
\hline 1 & 7 & $21.8^{\circ}$ & 0.652 \\
2 & 19 & $13.2^{\circ}$ & 1.068 \\
3 & 37 & $9.4^{\circ}$ & 1.489 \\
4 & 61 & $7.3^{\circ}$ & 1.912 \\
5 & 91 & $6.0^{\circ}$ & 2.347 \\
6 & 127 & $5.1^{\circ}$ & 2.759 \\
\hline \hline
\end{tabular}

In the transport simulations, the system is modeled by a tight-binding Hamiltonian

$$
H=\sum_{i} \epsilon_{i} c_{i}^{\dagger} c_{i}+\sum_{i j} t_{i j} c_{i}^{\dagger} c_{j} .
$$

The on-site energies are set to zero, as in defect-free graphene, or we include Anderson disorder by setting $\epsilon_{i}$ to random numbers uniformly distributed in the range $[-W / 2, W / 2]$, where $W$ is the disorder strength. The atomic positions determine the hopping elements $t_{i j}$ through an approximative formula for $\pi$-orbital overlap at different carbon sites $j$ and $i$ separated by $\mathbf{R}_{j}-\mathbf{R}_{i}=\mathbf{r}=(x, y)^{T}$,

$$
t_{i j}=t(\mathbf{r})=-\gamma_{0} e^{-\lambda\left(r-a_{c c}\right)},
$$

where $\gamma_{0}$ is the nearest-neighbor hopping parameter, $a_{c c}$ is the carbon-carbon distance, and the exponent is $\lambda \approx 3 / a_{c c}$. The formula in Eq. (2) is applied for atomic distances $r=|\mathbf{r}|$ reaching a cutoff $R_{c}$, beyond which $t_{i j}=0$. For the present problem, a good description is obtained for $R_{c} \approx 2 a_{c c}$. Going beyond the nearest-neighbor approximation means that the Dirac point in the band structure obtained from the model in Eq. (1) is shifted from zero to a higher energy, approximately equal to three times the next-nearest-neighbor hopping parameter obtained from Eq. (2), $E_{\text {Dirac }} \approx 0.33 \gamma_{0}$. The applied magnetic field modifies the hopping parameters through a Peierls phase,

$$
t_{i j} \rightarrow \exp \left[-\frac{i e}{\hbar} \int_{\mathbf{R}_{j}}^{\mathbf{R}_{i}} \mathbf{A} \cdot \mathbf{d l}\right] t_{i j} .
$$

The magnetic field enters naturally as an applied magnetic flux per hexagon (hexagon area of perfect graphene) in units of the flux quantum $\Phi_{0}=h / e$, where $h$ is Planck's constant and $e$ is the elementary charge. The magnetic field defines the magnetic length through $\ell_{B}=\sqrt{\hbar /(e B)}$, which in all simulations is smaller than the ribbon width, which means that the spectrum is dominated by Landau levels $E_{n}-E_{\text {Dirac }}=\sqrt{2 n} \hbar v_{f} / \ell_{B}=$ $\sqrt{n} \omega_{c}$, where $n \geqslant 0$ is an integer and $v_{f}$ if the Fermi velocity in the absence of magnetic field. In the ribbon geometry, Landaulevel bands acquire dispersive parts corresponding to the wellknown edge states which carry the current in the quantum Hall regime (see, for instance, Fig. 2 in Ref. [25] for an illustration).

In a magnetic field the two-terminal conductance equals the transverse conductivity $\sigma_{x y}$ in a Hall bar geometry when the contacts are perfect (as is the case in these simulations). The two-terminal conductance at zero temperature $G=G_{0} T(E)$ is given in terms of the conductance quantum $G_{0}=2 e^{2} / h$ and the linear response transmission function $T(E)$. The latter is computed through the retarded propagator of the system $G_{i j}^{R}(E)$ and self-energies of the lead surfaces $\Sigma_{\ell}^{R}(E)$, which remain after the leads have been eliminated in favor of the system in a standard way [29]. The leads are enumerated by the index $\ell$ ( $\ell=1$ and 2 for source and drain). The formula for the transmission is then

$$
T(E)=\operatorname{Tr}\left[\Gamma_{1}(E) G_{12}^{R}(E) \Gamma_{2}(E) G_{21}^{A}(E)\right],
$$

where $\Gamma_{\ell}=i\left[\Sigma_{\ell}^{R}-\left(\Sigma_{\ell}^{R}\right)^{\dagger}\right]$ and $G_{12}^{R}$ symbolizes the propagator between leads 1 and 2 . The advanced propagator $G_{21}^{A}(E)$ is the Hermitian conjugate of the retarded propagator, and the trace is over the surface sites. 
For local current flow patterns we need the lesser Green's function $G^{<}$. In the absence of electron correlations, the lesser Green's function is reduced to the form

$$
G_{i j}^{<}(E)=\sum_{\ell} f_{\ell}(E) \sum_{c \tilde{c}} G_{i c}^{R}(E)\left[\Gamma_{\ell}(E)\right]_{c \tilde{c}} G_{\tilde{c} j}^{A}(E),
$$

which involves the distribution functions of the leads $f_{\ell}(E)$. Surface sites of the leads are labeled by $c$ and $\tilde{c}$. Local charge current flow in the device (bond current between sites $i$ and $j$ ) is then written as

$$
\begin{aligned}
I_{i j} & =\frac{e}{\hbar} \int_{-\infty}^{\infty}\left[t_{i j} G_{j i}^{<}(E)-t_{j i} G_{i j}^{<}(E)\right] d E \\
& =\int_{-\infty}^{\infty} I_{i j}(E) d E .
\end{aligned}
$$

The retarded Green's function $G_{i j}^{R}(E)$ of the system is computed numerically through our own implementation of a recently developed recursive algorithm [30] within which sites are added one by one. Below we present the spectral current flow pattern $I_{i j}(E)$ assuming zero temperature and current injection from one contact.

\section{RESULTS}

In Fig. 2(a) we display a narrow nanoribbon with a $\Sigma=7$ $(m=1)$ grain boundary with ten 5-7 ring defect complexes. In a strong magnetic field, the current enters, for instance, from the top left corner and flows along the edge. Without scattering against defects, as in Fig. 2(a), the current reaches the right contact and is absorbed. Depending on the electron density, i.e., the location of the Fermi energy, we have a different number of Landau levels occupied and the corresponding number of edge states. Each edge state carries a unit of conductance including spin degeneracy $G_{0}$ since we neglect the Zeeman effect. The $n=0$ level is special in that valley degeneracy in the bulk is broken in the finite-size ribbon for the dispersive (edge state) parts of the spectrum. Higher Landau levels have valley degenerate edge states. The conductance sequence at zero temperature is therefore $G=(2 n+1) G_{0}$ for energies above the Dirac point (electron doping), with the same sequence for increasing hole doping. This sequence of plateaux is illustrated in Fig. 2(b) by the orange dashed line for electron doping (energies $E>E_{\text {Dirac }} \approx 0.33 \gamma_{0}$ ).

Let us now discuss the influence of the grain boundaries. It is well known that at each 5-7 defect, there are defect states. They may hybridize along the grain boundary, which then becomes metallic, as has been discussed in several papers [20-24]. In a magnetic field, with the grain boundary connecting the upper and lower edges, the question of what will happen with the edge-state current flow arises. In Fig. 2(b) we present the conductance as a function of energy, corresponding to the Fermi energy at zero temperature (which can be controlled by a back gate in a real device). For grain boundaries with large misorientation angles, for instance, $\theta=21.8^{\circ}(\Sigma=7)$ and $13.2^{\circ}(\Sigma=19)$ in Fig. 2(b), the metallicity of the grain boundary is apparent as the plateaux are not quantized. Edge states at opposite edges (which carry current in opposite directions) are connected by the metallic grain boundary which causes partial reflection of current and destruction
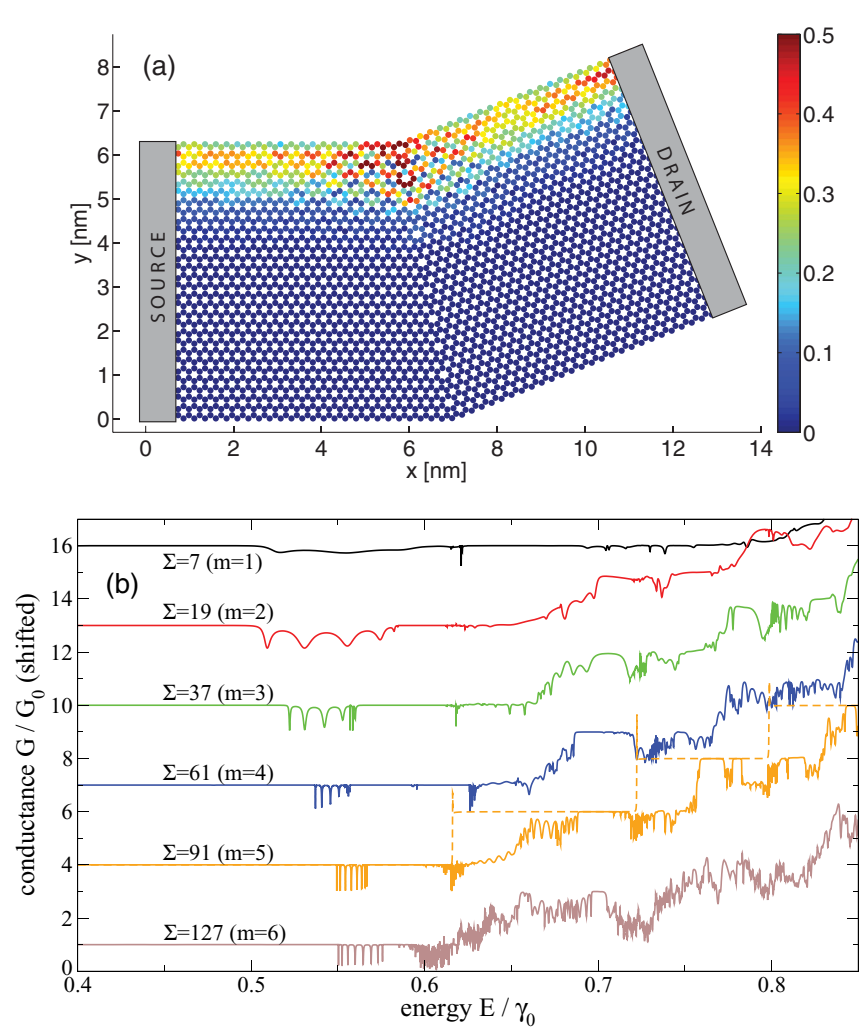

FIG. 2. (Color online) (a) A nanoribbon with a $\Sigma=7(m=1)$ grain boundary. Current flows along the upper edge (color scale denotes the absolute value of the current in units of $G_{0} V$, where $V$ is the applied voltage between source and drain) at energy $E=0.45 \gamma_{0}$ for which the conductance is quantized, $G=G_{0}$. (b) Conductance at zero temperature for nanoribbons with one grain boundary including ten defects as a function of energy. The different curves (shifted by $3 G_{0}$ for clarity) correspond to different grain-boundary angles and therefore also different ribbon widths since the defect density varies with grain-boundary angle. The dashed orange line is the quantized conductance of an ideal ribbon without grain boundary shifted by $3 G_{0}$. The magnetic field corresponds to a flux $\Phi=0.01 \Phi_{0}$ per hexagon in all cases. In (a) the Anderson disorder strength is $W=0.25 \gamma_{0}$, while it is $W=0$ in (b).

of the plateaux. For lower grain-boundary angles, the 5-7 defects are farther apart, and hybridization is less effective. In a magnetic field, a more pronounced reflection resonance pattern then develops; see, for instance, the $\Sigma=127(m=6)$ grain-boundary conductance curve in Fig. 2(b) (brown line). This resonance behavior is particularly clear for the $n=0$ plateau, where a comb of resonances is well defined near $E \approx 0.56 \gamma_{0}$. The number of resonances (size of the comb) depends on the number of defects along the grain boundary. In Fig. 2(b) the ribbon width is varying so that the grain boundaries always hold ten 5-7 defects. The comb is present for all grain-boundary misorientation angles, but the enhanced hybridization and enhanced metallicity for grain boundaries with more dense defect densities is clear in Fig. 2(b) when comparing the combs' shapes for grain boundaries with decreasing $m$. For higher energies, many more resonances appear, and quantization for higher Landau levels is completely destroyed. 


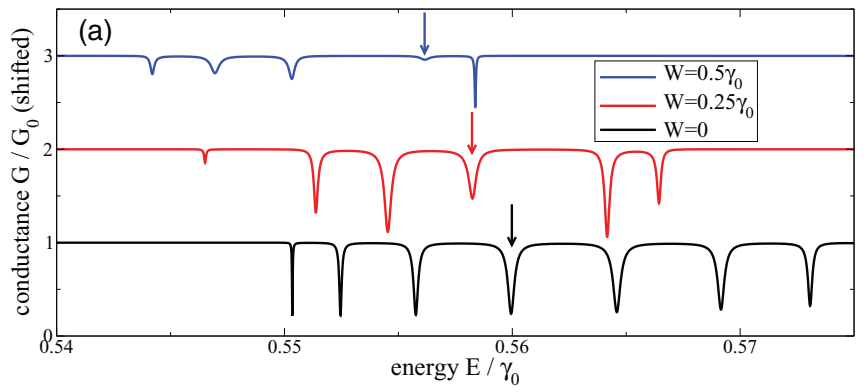

x-component of bond-currents
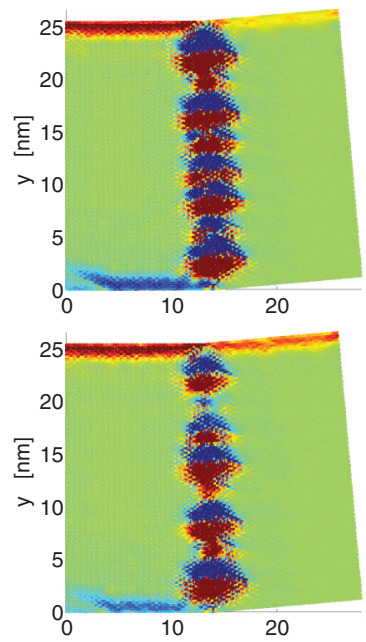

(c)
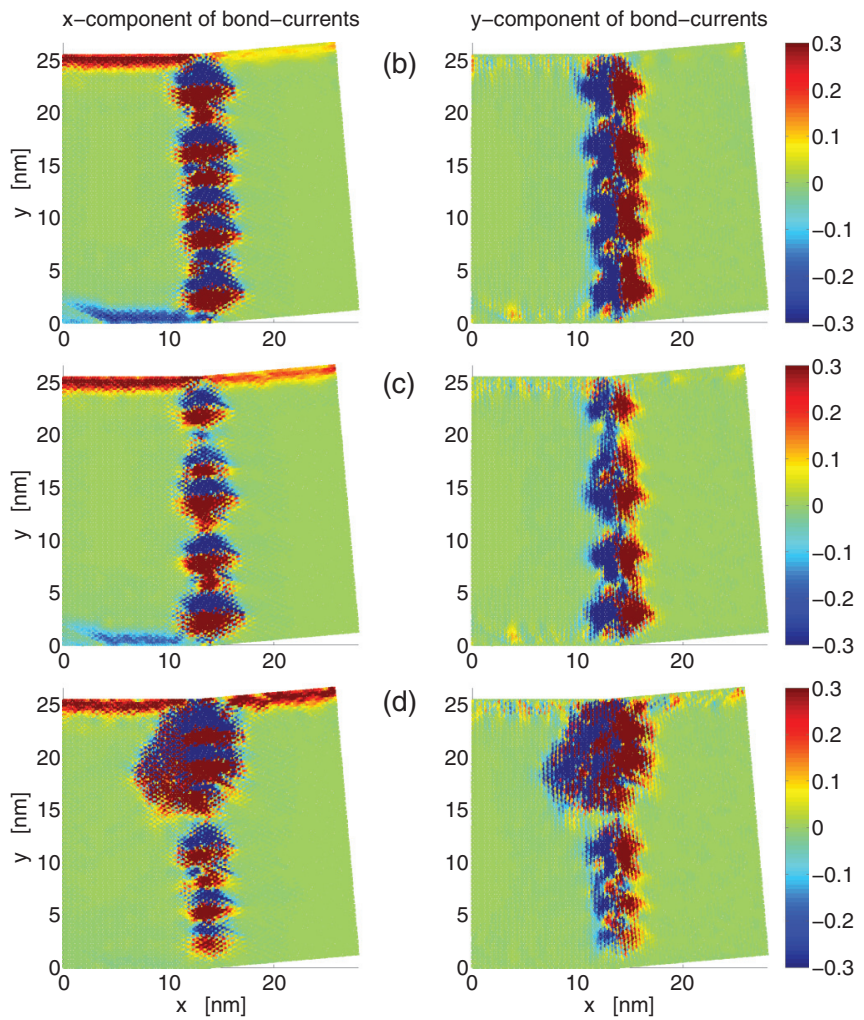

(d)

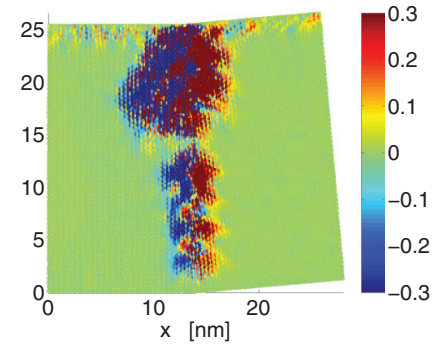

FIG. 3. (Color online) (a) Evolution of the conductance in Fig. 2(b) for the $\Sigma=127$ ( $m=6)$ grain boundary with increasing Anderson disorder strength $W$ (other model parameters are held fixed). Examples of local current flow patterns near the grain boundary for (b) $W=0$, (c) $W=0.25 \gamma_{0}$, and (d) $W=0.5 \gamma_{0}$. The energies where these current flow patterns appear are marked in (a) by vertical arrows. Disorder is distributed randomly across the whole displayed systems, with ideal source and drain contacts attached to the left and right ribbons.

We can gain additional insight into the nature of the conductance fluctuations by looking at the local current flow patterns. In Fig. 3(a) we display a zoom of the resonance comb on the $n=0$ plateau for the $\Sigma=127(m=6)$ grain boundary (black curve for $W=0$ ). For the energy indicated by the vertical black arrow, we present the local current flow pattern in Fig. 3(b). The current flows in a circular fashion around each 5-7 ring defect and at the same time displays an envelope pattern across the entire grain boundary from the upper to the lower ribbon edges. The envelope contains a varying number of nodes for the different resonances in the comb. Each resonance, therefore, corresponds to a particular hybridization of the defect states along the grain boundary.

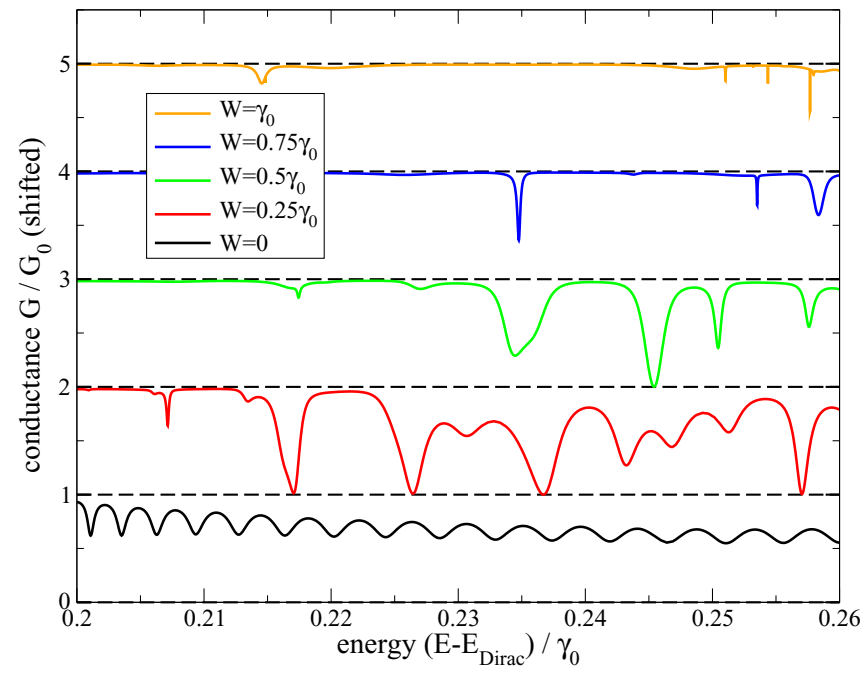

FIG. 4. (Color online) Conductance on the lowest plateau at zero temperature for a 50-nm-wide nanoribbon with a $\Sigma=7(m=1)$ grain boundary for varying Anderson impurity disorder strength. The different curves are shifted by $G_{0}$ for clarity, and the magnetic field corresponds to a flux $\Phi=0.01 \Phi_{0}$ per hexagon.

In Fig. 3(a), we study the evolution of the resonance comb with increasing Anderson disorder strength $W$. For $W=0.25 \gamma_{0}\left(\sim 0.68 \mathrm{eV}\right.$ for $\left.\gamma_{0} \approx 2.7 \mathrm{eV}\right)$, the resonances are shifted, and some are weakened. For $W=0.5 \gamma_{0}$ this effect is more pronounced, and at higher $W$ resonances disappear. The current flow patterns for the conductance dips marked by the red arrow $\left(W=0.25 \gamma_{0}\right)$ and the blue arrow $\left(W=0.5 \gamma_{0}\right)$ are shown in Figs. 3(c) and 3(d), respectively. For increasing disorder strength, the current flows chaotically down the grain boundary, but eventually [for strong $W$, Fig. 3(d)] a situation resembling localization along the grain boundary appears, and quantization is improved. For wider ribbons, a weaker $W$ is enough for recovery of the first plateau. This picture of localization agrees with the recent results in Refs. $[10,15]$ for the special 5-8-5 line defect mentioned in the Introduction.

In Fig. 4, we study the effect of Anderson disorder for a 50 -nn-wide ribbon with a $\Sigma=7(m=1)$ grain boundary. This grain boundary has the most dense defect density and displays for $W=0$ nonquantized conductance, reflecting the metallicity of the grain boundary (black wavy curve). Introducing weak Anderson disorder, $W=0.25 \gamma_{0}$, leads to development of more sharp resonances (dips with zero conductance; red curve). For larger $W$, also, these resonances disappear. The sensitivity of resonances to disorder depends on the grain-boundary angle. Smaller grain-boundary angles correspond to less defect density, sharper resonances, and higher sensitivity to disorder. For system sizes that we have considered, higher plateaux are, however, always destroyed, while the first plateau is more robust. This corresponds to the typical experimental situation where the higher plateaux $(n \geqslant 1)$ are, in general, more difficult to observe. Finally, we expect that for larger system sizes (wider ribbons), smaller values of the disorder strength $W$ will lead to recovery of the plateau since the localization length increases with decreasing $W$ in general. A more in-depth description of the localization 
properties of the grain-boundary states in a magnetic field is beyond the scope of this paper.

\section{SUMMARY AND OUTLOOK}

In real materials, the grain boundary may not be as perfect as considered in this paper. There may be many locally well defined and ordered grain boundaries (for instance, of the [0001] tilt angle form considered here), but on a larger scale they may form a complicated grain-boundary network across the device. In this paper we have focused on the perfectly ordered grain boundary in order to study theoretically basic properties and major trends. In a more disordered network the hybridization of dislocation core electronic states into metallic bands may be altered and also become more sensitive to disorder. We leave for the future a more thorough study of this more complicated situation. A hint can be found in Ref. [15], in which a wave propagation method was used to study localization inside grains in the presence of a magnetic field. It was shown that the magnetic length should be smaller than the grain radius in order for Landau levels to be formed locally inside the grain. It remains for future work to study quantum transport in a restricted geometry (with edge states) including more general types of grain-boundary networks.
In conclusion, we have studied the influence of [0001] tilt grain boundaries on the quantum Hall effect in granular graphene. We find that electronic states formed at dislocation cores (5-7 complexes) in the grain boundary form metallic bands that in a magnetic field can short-circuit counterpropagating edge states on opposite sides of the Hall bar. The QHE is thereby destroyed. Depending on the defect density along the grain boundary, weak Anderson disorder can lead to recovery of at least the $n=0$ plateau. This indicates that the reason why the QHE so far has not been observed to metrological accuracy in graphene made by CVD on metal substrates could be due to the granularity of this material. One way to test this hypothesis experimentally is to manufacture two different types of Hall bars with well-defined geometries: one with and one without a single grain boundary connecting the two sides of the Hall bar with counterpropagating edge states. This geometry would be analogous to the one studied in Refs. [25,26], in which bilayer stripe defects were shown to be detrimental to the QHE.

\section{ACKNOWLEDGMENTS}

We acknowledge financial support from the European Union seventh framework program through the STREP ConceptGraphene, the Swedish Foundation for Strategic Research, and the Knut and Alice Wallenberg foundation.
[1] K. S. Novoselov, A. K. Geim, S. V. Morozov, D. Jiang, M. I. Katsnelson, I. V. Grigorieva, S. V. Dubonos, and A. A. Firsov, Nature (London) 438, 197 (2005).

[2] Y.Zhang, Y.-W. Tan, H. L. Stormer, and P. Kim, Nature (London) 438, 201 (2005).

[3] A. Tzalenchuk, S. Lara-Avila, A. Kalaboukhov, S. Paolillo, M. Syväjärvi, R. Yakimova, O. Kazakova, T. Janssen, V. Falko, and S. Kubatkin, Nat. Nanotechnol. 5, 186 (2010).

[4] T. J. B. M. Janssen, N. E. Fletcher, R. Goebel, J. M. Williams, A. Tzalenchuk, R. Yakimova, S. Kubatkin, F. Lara-Avila, and V. I. Fal'ko, New J. Phys. 13, 093026 (2011).

[5] T. J. B. M. Janssen, J. M. Williams, N. E. Fletcher, R. Goebel, A. Tzalenchuk, R. Yakimova, S. Lara-Avila, S. Kubatkin, and V. I. Fal'ko, Metrologia 49, 294 (2012).

[6] W. Poirier, F. Schopfer, J. Guignard, O. Thévenot, and P. Gournay, C. R. Phys. 12, 347 (2011)

[7] F. Lafont, R. Ribeiro-Palau, D. Kazazis, A. Michon, O. Couturaud, C. Consejo, T. Chassagne, M. Zielinski, M. Portail, B. Jouault, F. Schopfer, and W. Poirier, Nat. Commun. 6, 6806 (2015).

[8] T. Shen, W. Wu, A. Yu, C. A. Richter, R. Elmquist, D. Newell, and Y. P. Chen, Appl. Phys. Lett. 99, 232110 (2011).

[9] Y. Nam, J. Sun, N. Lindvall, S. J. Yang, D. Kireev, C. R. Park, Y. W. Park, and A. Yurgens, Appl. Phys. Lett. 103, 233110 (2013).

[10] F. Lafont, R. Ribeiro-Palau, Z. Han, A. Cresti, A. Delvall'ee, A. W. Cummings, S. Roche, V. Bouchiat, S. Ducourtieux, F. Schopfer, and W. Poirier, Phys. Rev. B 90, 115422 (2014).

[11] A. C. Ferrari et al., Nanoscale 7, 4598 (2015).
[12] F. Banhart, J. Kotakoski, and A. V. Krasheninnikov, ACS Nano 5, 26 (2011).

[13] O. V. Yazyev and Y. P. Chen, Nat. Nanotechnol. 9, 755 (2014).

[14] H.-B. Yao, X.-L. Lü, and Y.-S. Zheng, Phys. Rev. B 88, 235419 (2013).

[15] A. W. Cummings, A. Cresti, and S. Roche, Phys. Rev. B 90, 161401(R) (2014).

[16] P. Y. Huang, C. S. Ruiz-Vargas, A. M. van der Zande, W. S. Whitney, M. P. Levendorf, and J. W. Kevek et al., Nature (London) 469, 389 (2011).

[17] K. Kim, Z. Lee, W. Regan, C. Kisielowski, M. F. Crommie, and A. Zettl, ACS Nano 5, 2142 (2011).

[18] J. An, E. Voelk1, J. W. Suk, X. Li, C. W. Magnuson, L. Fu, P. Tiemeijer, M. Bischoff, B. Freitag, E. Popova, and R. S. Ruoff, ACS Nano 5, 2433 (2011).

[19] P. Nemes-Incze, P. Vancso, Z. Osvath, G. I. Mark, X. Jin, Y.-S. Kim, C. Hwang, P. Lambin, C. Chapelier, and L. P. Biro, Carbon 64, 178 (2013).

[20] O. V. Yazyev and S. G. Louie, Nat. Mater. 9, 806 (2010).

[21] J. Lahiri, Y. Lin, P. Bozkurt, I. I. Oleynik, and M. Batzill, Nat. Nanotechnol. 5, 326 (2010).

[22] O. V. Yazyev, Solid State Commun. 152, 1431 (2012).

[23] S. Ihnatsenka and I. V. Zozoulenko, Phys. Rev. B 88, 085436 (2013).

[24] F. Gargiulo and O. V. Yazyev, Nano Lett. 14, 250 (2014)

[25] T. Lofwander, P. San-Jose, and E. Prada, Phys. Rev. B 87, 205429 (2013). 
[26] T. Yager, A. Lartsev, S. Mahashabde, S. Charpentier, D. Davidovikj, A. Danilov, R. Yakimova, V. Panchal, O. Kazakova, A. Tzalenchuk, S. Lara-Avila, and S. Kubatkin, Nano Lett. 13, 4217 (2013).

[27] J. M. Carlsson, L. M. Ghiringhelli, and A. Fasolino, Phys. Rev. B 84, 165423 (2011).
[28] MATERIALS STUDIO, release 7.0, Accelrys Software Inc., San Diego, CA, 2013.

[29] S. Datta, Electronic Transport in Mesoscopic Systems (Cambridge University Press, Cambridge, 1997).

[30] K. Kazymyrenko and X. Waintal, Phys. Rev. B 77, 115119 (2008). 